\title{
Il y a cinquante ans : les premières réactions de fusion nucléaire induites par laser
}

Jean-Louis Bobin (jean-louis.bobin@sorbonne-universite.fr)

Professeur émérite à Sorbonne Université

Peu après l'invention des lasers au début des années 1960, des calculs d'ordre de grandeur montraient leur intérêt pour la fusion nucléaire. En même temps, débutaient discrètement des programmes de recherches dans des laboratoires à vocation militaire. C'est ainsi qu'au centre de Limeil de la Direction des Applications Militaires du Commissariat à l'Énergie Atomique (CEA-DAM) fut lancée l'étude des plasmas créés par laser, en coopération avec les Laboratoires de Marcoussis de la Compagnie Générale d'Électricité (CGE).

Cet effort, dont l'histoire est relatée dans cet article, a abouti à un résultat spectaculaire pour l'époque (1969) une émission de neutrons contrôlable.

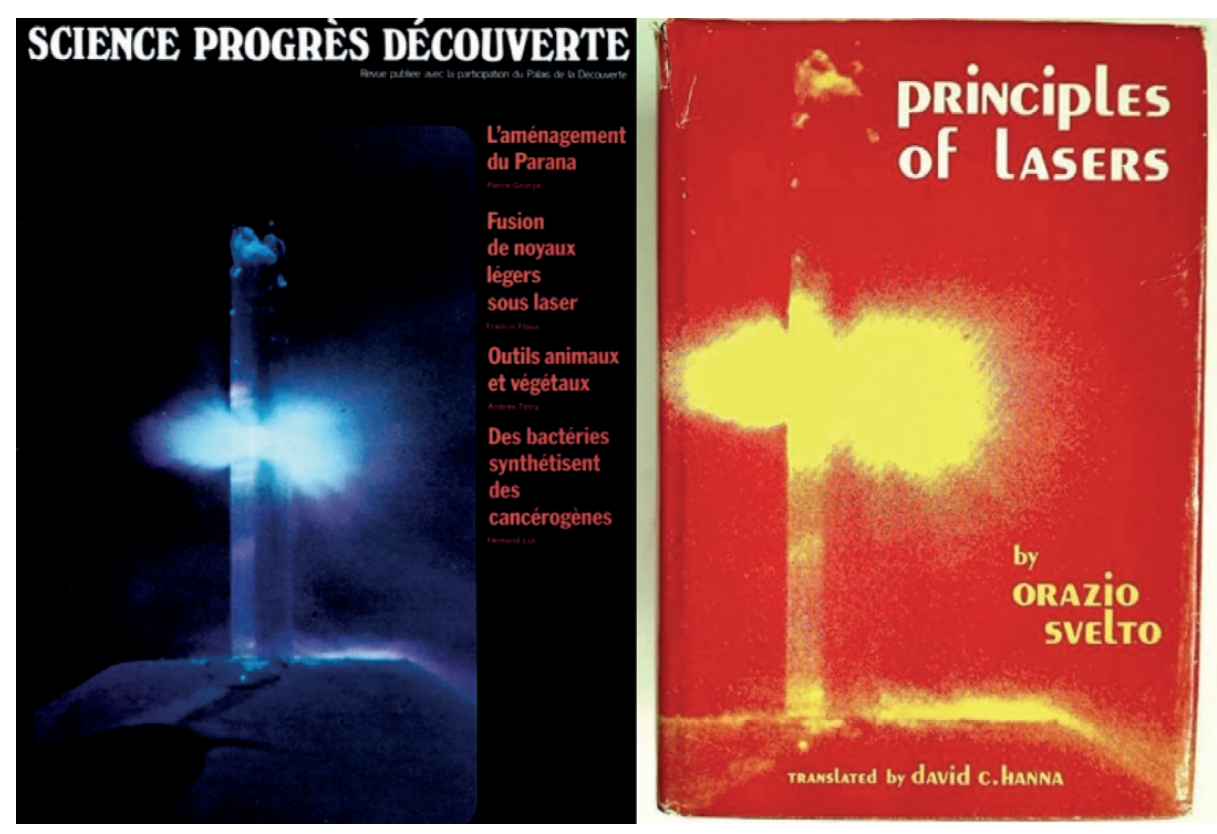

Deux exemples des suites d'une communication naïve (voir p. 25). La photo remise à la presse d'un bâtonnet de deutérium solide irradié par laser ne précisait pas le sens vertical (comparer avec la figure 3b, p. 24). Les journalistes ont reproduit ce qu'ils avaient vu.

\section{La fusion nucléaire} par laser, une idée dans l'air des années 1960

Matérialisation d'un vieux rêve de l'humanité, l'amplification de la lumière, le laser, inventé en 1960 [1], fut d'abord un simple objet de curiosité : "solution sans problème " disaient les persifleurs de service. On se mit donc à la recherche de problèmes. Parmi tous les domaines qui s'ouvrirent alors, la fusion nucléaire fit l'objet d'un développement particulièrement dynamique. Les fortes intensités lumineuses qu'il était envisageable d'atteindre à partir de lasers laissaient en effet entrevoir la possibilité d'obtenir, par conversion d'énergie lumineuse en énergie thermique, les conditions nécessaires à une combustion thermonucléaire des isotopes lourds de l'hydrogène, deutérium et tritium. Le laser pouvait-il apporter une alternative crédible au confinement magnétique, seule voie explorée jusque-là et qui alors marquait le pas ? En l'espace de quelques mois, aux États-Unis, en Europe et dans ce qui était alors l'URSS, on effectua les premiers calculs. Il apparut qu'avec des hypothèses raisonnables sur la dynamique du procédé, des conditions d'intérêt thermonucléaire sont atteintes [2]. 
$>>$

L'histoire eut aussi une face cachée. À Livermore, laboratoire californien spécialisé dans les programmes thermonucléaires, des experts de la physique des armes [3] utilisèrent leurs connaissances pour élaborer, à l'aide de codes numériques, des schémas qui entrent aujourd'hui dans la catégorie connue sous le nom de "Fusion par Confinement Inertiel " (FCI). Dans les années 1960, de telles idées relevaient du secret le plus absolu et ne furent donc pas rendues publiques. Un modeste programme expérimental fut lancé à partir de 1962.

Aux États-Unis comme en France, l'histoire de la fusion par laser pendant cette période est mal connue ${ }^{(a)}$. Pour la France, on trouve quelques indications sous la forme de souvenirs personnels dont Jean Robieux (voir l'encadré ci-dessous), alors figure de proue des Laboratoires de Marcoussis appartenant au groupe de la Compagnie Générale d'Électricité $(\mathrm{CGE})^{(b)}$, a parsemé ses ouvrages [4]. Il est peu loquace sur la partie CEA des travaux exécutés en collaboration avec le Centre d'Études de Limeil.

\section{Aux origines}

Exception parmi les chercheurs ayant réagi à l'invention des lasers en pensant à la fusion nucléaire, Jean Robieux travaillait dans un environnement industriel. Il était chargé, à la tête du département de Recherches Physiques de Base des Laboratoires de Marcoussis, de mettre en œuvre des innovations susceptibles d'ouvrir des marchés à des produits de haute technologie fabriqués et commercialisés par le groupe CGE. Il fut immédiatement séduit par les potentialités des lasers et proposa d'en développer les applications dans des domaines aussi variés que les télécommunications (un des métiers de base de la CGE), la séparation isotopique ou encore la fusion thermonucléaire.

Robieux attaqua le problème de la fusion par laser avec des idées qui aujourd'hui paraissent bien naïves. Mais, à l'époque, elles ont permis un utile débroussaillage, grâce à quelques calculs d'ordre de grandeur. On connait depuis les années 1950 les conditions favorables à la fusion thermonucléaire $^{(\mathrm{c})}$. Transposées au cas de la lumière laser irradiant une cible, elles se traduisent par une triple exigence : être capable d'ioniser, de chauffer jusqu'à $10^{8} \mathrm{~K}$, et que la réaction deutérium-tritium ait lieu

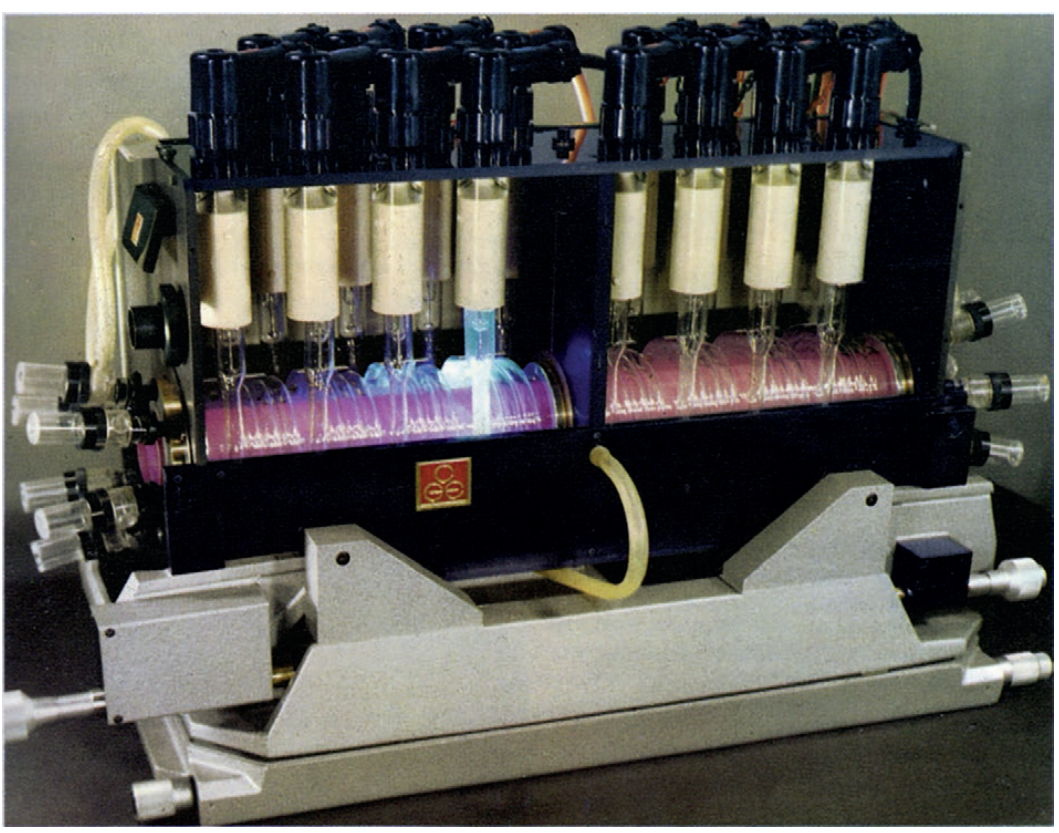

1. Amplificateur de puissance de la CGE : standard des années 1960 pour un barreau de verre au néodyme de diamètre $5 \mathrm{~cm}$. pendant un temps $\tau$ suffisant pour satisfaire au critère de Lawson : n $\tau \geq 10^{14} \mathrm{~cm}^{-3}$.s, où $\mathrm{n}$ est la densité des ions (nombre par $\mathrm{cm}^{3}$ ). À l'état solide, la densité ionique est d'environ $510^{22} \mathrm{~cm}^{-3}$, ce qui impose un temps de confinement supérieur à 2 nanosecondes (ns). Il faut compter sur l'inertie de la matière même chaude pour que la densité du solide soit maintenue au moins pendant ce temps.

Les lasers de 1961-62 étaient de toutes premières réalisations aux performances modestes, une fraction de joule en 100 ns. Les chiffres obtenus par Robieux indiquaient l'effort minimal à produire en matière de technologie laser pour pouvoir réaliser les conditions de la fusion nucléaire. Alertée, la direction de la CGE réagit favorablement à l'idée que le potentiel de recherche et de développement de l'entreprise permettait de se lancer dans l'étude et la réalisation de lasers plus puissants et aux durées d'impulsion plus courtes qu'on n'avait su le faire jusqu'alors.

Selon une tradition bien française, la CGE avait ses relais au plus haut niveau gouvernemental. Des rapports [5] firent, fin 1962 et début 1963, leur chemin jusqu'au sommet de l'État. Confidentiels, ils ne furent pas publiés dans la littérature scientifique, empêchant ainsi Jean Robieux de figurer parmi les pères fondateurs reconnus de la fusion nucléaire par laser.

\section{Jean Robieux}

Ancien élève de l'École polytechnique, docteur es-sciences et titulaire d'un Master of Sciences du California Institute of Technology (Caltech), Jean Robieux (1925-2012) fut l'un des principaux spécialistes français du laser et de l'optronique. Il est à l'origine de la découverte du principe du contrôle de la fusion nucléaire par laser, puis du principe de la séparation isotopique par laser.

Il a été président de la Société d'encouragement pour l'industrie nationale, et membre des Académies des sciences et des technologies.

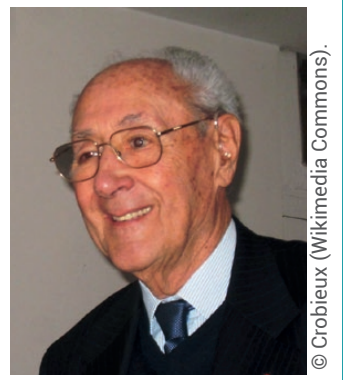

Jean Robieux en 2010 


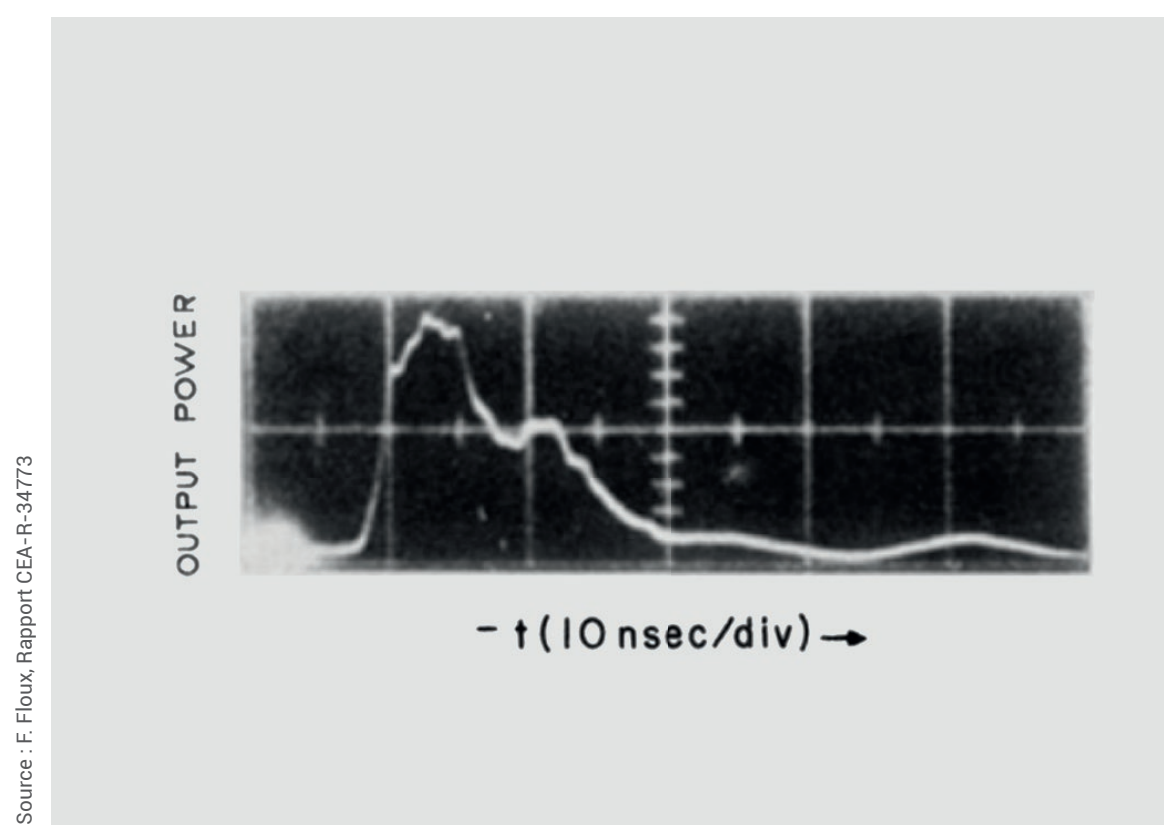

2. Impulsion laser mise en forme après découpage. En 1969, la puissance en bout de chaine ("output power") pouvait atteindre $7 \mathrm{GW}$.

Cependant, ses idées donnèrent lieu à un dépôt de brevets d'invention publiés au Bulletin officiel de la propriété industrielle [6]. Sous le même titre : "Dispositif de fusion thermonucléaire commandée ", ils ont été soumis en 1963 par la CGE, mais ne furent officiellement accordés qu'en juin 1968, après un long délai justifié par le caractère de leur contenu considéré pendant tout ce temps comme sensible.

Le lobbying exercé par la CGE se traduisit par le financement d'un programme de recherches sur la fusion par laser mené en commun avec le CEA. Par l'intermédiaire de la Direction des Recherches et Moyens d'Essais (DRME) du ministère des Armées, les Laboratoires de Marcoussis recevaient une dotation pluriannuelle pour développer des lasers de puissance. La partie interaction avec des cibles et fusion nucléaire était confiée au centre de Limeil de la Direction des Applications Militaires (DAM) du CEA, où les personnels avaient la compétence requise en matière de recherches thermonucléaires, avec l'avantage de travailler au sein d'un environnement où la discrétion est le plus souvent impérative. Le futur ministre $^{(\mathrm{d})}$ Pierre Aigrain, alors directeur à la DRME, fit beaucoup pour cet arrangement.

\section{Des lasers de plus en plus puissants}

Le programme de recherches entrepris à Limeil utilisait pour l'essentiel des lasers conçus et réalisés par le partenaire institutionnel, la CGE, à partir de cahiers des charges élaborés en commun.

Grâce aux efforts conjugués des deux parties, on assista à une progression spectaculaire des performances, de l'encombrement et... du cout des lasers de puissance à impulsion géante. Bien au-delà du modeste oscillateur à rubis ou à verre des débuts, on aboutit à des ensembles imposants qu'on imaginait, avec raison comme le prouva la suite, presque indéfiniment extensibles. Leur architecture générale devenue un standard, comportait un étage pilote où se formait l'impulsion dans un barreau oscillateur en cavité. L'impulsion traversait ensuite une cascade d'amplificateurs dont le diamètre des barreaux allait croissant. L'augmentation du diamètre du faisceau était assurée par des systèmes afocaux insérés entre les étages d'amplification.

Le matériau actif des lasers construits par la CGE était le verre au néodyme émettant dans l'infrarouge à une longueur d'onde de $1 \mu \mathrm{m}$. Ce matériau permettait de se fournir chez des industriels hexagonaux héritiers d'une longue tradition d'excellence.
Dans les amplificateurs (fig. 1), le barreau de verre était entouré d'une lampe éclair ("flash ») hélicoïdale disposée à l'intérieur d'un réflecteur métallique. Le diamètre des barreaux de verre ne peut pas croitre audelà d'une dizaine de centimètres, soit le double de la longueur d'absorption de la lumière des flashs. Or l'intensité lumineuse doit rester inférieure à une valeur garantissant l'absence de dommages. Il existait ainsi une double limitation (toujours présente sur les instruments des générations suivantes) : le diamètre d'un faisceau et la puissance qu'il peut transporter.

Les perfectionnements apportés aux lasers de puissance eurent pour effet de porter à 500 joules l'énergie contenue dans une impulsion lumineuse de 30 nanosecondes, démonstration faite à Marcoussis en 1967 [7]. Par ses réalisations, la CGE se trouvait à cette date largement en tête de la course à la puissance. Les chercheurs de Limeil avaient ainsi la possibilité de devancer leurs concurrents.

La tendance générale qui se dégageait des expériences d'interaction était qu'il fallait aller vers des impulsions laser, certes plus puissantes mais aussi plus courtes que les 30 ns à mi-hauteur fournies par l'oscillateur de base, quelle que soit la méthode de déclenchement. Le front de montée devait être raidi sur plusieurs ordres de grandeur d'intensité. Autour de 1967-1968, on plaça une cellule appelée cellule de Pockels en sortie de pilote. Grâce à une électronique ad hoc, elle découpait dans l'impulsion géante, trop longue, une forme mieux adaptée aux demandes (fig. 2). Pour compenser partiellement la perte d'énergie due à la mise en forme, on ajouta un étage d'amplification.

La création de plasma au moyen d'un faisceau laser est une conséquence de la concentration d'énergie obtenue au foyer d'une lentille ou d'un miroir. La qualité de la focalisation est d'une extrême importance. Après divers tâtonnements, on adopta le principe d'une lentille plan-convexe dont la surface courbe était asphérique et calculée point par point [8].

Pour la fabrication, on s'adressa à l'Institut d'Optique où il existait une machine à commande numérique capable, à partir d'un tableau de données, de tailler le verre suivant n'importe quelle surface courbe. De magnifiques pièces d'optique dont le diamètre dépassait $10 \mathrm{~cm}$ furent ainsi réalisées. 


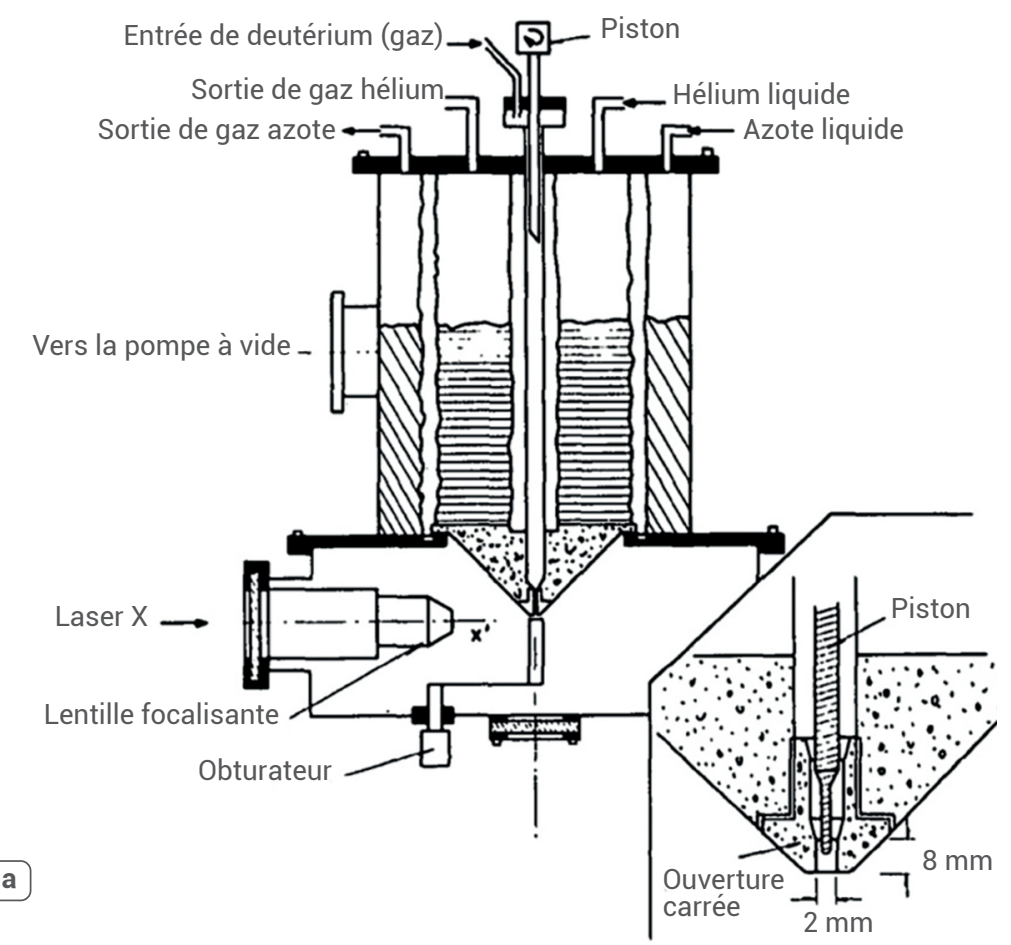

3. Le dispositif expérimental.

(a) Schéma du cryostat et (en bas à droite) détail de la filière d'extrusion [9].

(b) Impact d'un faisceau laser sur un glaçon de deutérium, extrudé du dispositif (a).

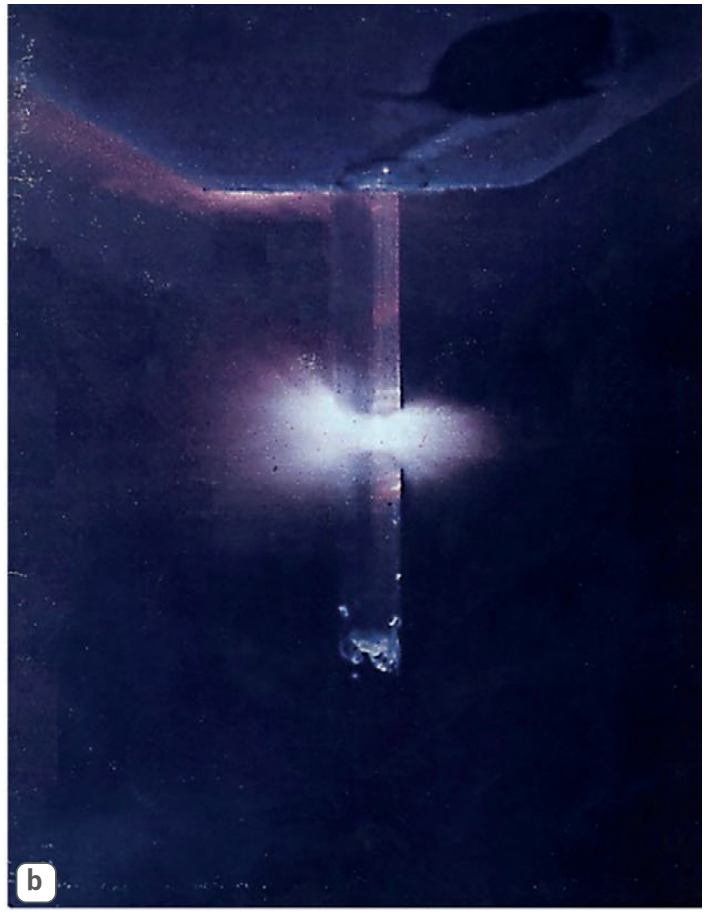

$\gg>$

\section{L'irradiation des solides}

Pour obtenir des réactions de fusion nucléaire, il fallait des cibles solides riches en isotopes lourds de l'hydrogène, essentiellement du deutérium, le tritium plus réactif étant cher, radioactif et de manipulation délicate. À Limeil, où l'on pratiquait des techniques de réfrigération jusqu'à la température de l'hélium liquide, le choix s'était porté sur le deutérium solide. Mais le pari était osé. Allait-on obtenir un solide avec des surfaces planes bien définies en vue de l'interaction ? Quel serait son comportement sous vide?

La méthode adoptée était simple : d'abord condensation du gaz dans un cryostat, solidification puis extrusion par le fond à travers une ouverture carrée, la pression étant exercée par un piston enfoncé manuellement au moyen d'une vis. Ce dispositif (fig. 3a) était superposé à une chambre d'interaction où régnait un vide secondaire. Il donna du premier coup d'excellents résultats [9]. Sans être tout à fait comparable à du dentifrice, le deutérium solide se révéla suffisamment plastique pour être extrudé, mais assez rigide pour garder sa forme sous vide en attendant le tir laser (fig. 3b). Le point de focalisation ne pouvait être éloigné de plus d'un centimètre du «nez » de la filière.

Les contraintes étaient sévères pour un ensemble où devaient cohabiter de très basses et de très hautes températures : les quelques kelvins de l'hélium liquide du cryostat et les millions de degrés du plasma d'interaction, celui-ci heureusement très localisé et transitoire.

\section{Les neutrons qui venaient du froid}

La préparation de bonnes cibles en deutérium étant assurée, les expériences d'interaction se sont déroulées de façon très méthodique. Elles étaient, à la fin de 1968, à la pointe de la physique et de la technique. Les mesures de la température du plasma en fonction de l'intensité laser laissaient espérer des réactions thermonucléaires deutérium-deutérium (D-D), aisément identifiables grâce aux neutrons de 2,45 MeV produits.

Ceux-ci étaient détectés de deux façons. D'abord par des scintillateurs plastiques d'assez grand volume pour suivre, avec une résolution comparable à celle des autres diagnostics, l'évolution temporelle de leur émission et mesurer leur énergie par temps de vol. Ensuite, par des compteurs au trifluorure de bore pour évaluer leur nombre.

Or, en 1968, des émissions de neutrons, mais sporadiques et faibles, avaient été annoncées par des chercheurs de l'institut Lebedev à Moscou. Une équipe de cet institut avait détecté des neutrons en irradiant avec des impulsions picoseconde des cibles deutérées.

À Limeil, les neutrons se firent attendre jusqu'au début de l'été 1969. Leur nombre, comptabilisé par les compteurs, était beaucoup plus important que chez les Russes : quelques milliers contre quelques dizaines. Ce résultat fut placé sous embargo, le temps qu'une étude systématique montre de façon indiscutable que cette émission était contrôlable.

À la fin du mois d'aout 1969, les certitudes étaient acquises : des neutrons pouvaient être produits dans des conditions prévisibles et reproductibles, en irradiant du deutérium solide avec les impulsions laser nanoseconde à front raidi. 


\section{Après le froid, le show}

Les hautes sphères du CEA furent aussitôt avisées du succès de ces expériences. Annonce fut alors faite urbi et orbi par l'intermédiaire d'un communiqué à l'agence France-Presse. Une note à l'Académie des sciences, transmise par le Haut-Commissaire Francis Perrin, était également prévue. Le retentissement hexagonal fut suffisamment grand pour obliger de réunir en toute hâte une conférence de presse à Limeil, qui se tint en fin d'après-midi du lundi 15 septembre 1969 , le même jour que la présentation à l'Académie.

Quelle aubaine! Un centre militaire du CEA s'ouvrait aux journalistes qui, connus ou obscurs, vinrent en foule. Les hiérarchies du CEA, de la DRME et de la CGE, étaient là aussi. Tous les visiteurs eurent droit, par groupes, à une démonstration de tir laser. Afin que l'ensemble des présents autour de la chambre d'interaction puisse voir l'expérience, on avait formé sur un écran, au moyen d'une lentille, une image agrandie mais renversée du glaçon de deutérium pendu à son porte-cible. Largement diffusée, l'image de l'irradiation d'un bâtonnet dressé sur un piédestal est entrée dans la légende (voir les couvertures de deux revues, reproduites p. 21, et comparer avec la figure $3 b$ ).

\section{Retombées}

Cet aboutissement de recherches menées depuis 1963 a marqué une étape importante dans le programme laser du CEA-DAM.

L'émission des neutrons fit l'objet de nombreux articles et rapports [10]. Au-delà de l'obtention de neutrons, de solides connaissances, tant théoriques que pratiques, avaient été acquises à la fin des années 1960 sur la dynamique des plasmas créés par impact laser. Combinées à la déclassification, en 1972, de la fusion inertielle par compression de granules jusqu'à des densités de l'ordre de 10000 fois celle du solide [11], elles ont contribué à lancer les développements qui ont conduit à la construction et à l'exploitation du laser mégajoule [12].

à travers une ouverture carrée, la pression étant exercée par un piston enfoncé manuellement...

À la fin du mois d'aout 1969 , les certitudes étaient acquises : des neutrons étaient produits dans des conditions prévisibles et reproductibles, en irradiant du deutérium solide avec les impulsions laser nanoseconde à front raidi. "

(a) Un historien des sciences du Stevens Institute of Technology, Alex Wellerstein, a effectué quelques recherches sur le sujet, limitées aux USA. Ses travaux n'ont pas été publiés mais présentés lors de diverses conférences, dont l'une à Paris le 17 décembre 2015. On peut lire un compte rendu de l'une de ces présentations sous le titre "The twisted tale of laser fusion" sur le blog de la journaliste scientifique Chelsey B. Coombs : http://scienceline.org/author/chelsey-b-coombs/.

(b) Futur Alcatel-Lucent, et aujourd'hui Nokia.

(c) Pour des généralités sur la fusion nucléaire, voir M. Decroisette, Reflets de la physique 21 (2010) 35-38 et J. Jacquinot, Reflets de la physique 32 (2013) 19-25.

(d) P.Aigrain a été président de la Société Française de Physique en 1986.

\section{Références}

1• T.H. Maiman, Nature 187 (1960) 493 : The Laser Odyssey, Laser Press (2000).

2• N. G. Basov et O. N. Krokhin, Soviet Physics JETP 19 (1964) 123 ; A. Kastler, C.R. Acad. Sc. Paris, 258 (1964) 489 ; J.M. Dawson, Phys. Fluids 7 (1964) 981.

3. R. E. Kidder, Laser Fusion: The First Ten Years 1962-1972, UCRL-BOOK-222681 (2006) ; J. H. Nuckolls, Contributions to the Genesis and Progress of ICF, UCRL-BOOK-219136 (2006).

4. J. Robieux, High Power Interactions, Éditions Lavoisier (2000); Vers l'énergie abondante sans pollution. La fusion nucléaire par laser, Éditions Louis de Broglie / Librairie Eyrolles (2008).

5. Pour des généralités sur la fusion nucléaire, voir M. Decroisette, Reflets de la physique 21 (2010) 35-38, et J. Jacquinot, Reflets de la physique 32 (2013) 19-25.
5. J. Robieux et R. Rocherolles, Production de plasmas très denses et très chauds à partir de l'état solide illuminé par un faisceau laser très puissant. Rapports CGE du 26 novembre 1962 et du 7 février 1963.

6. Bulletin officiel de la propriété industrielle, $\mathrm{n}^{\circ} 29$ (1968).

7• J. Robieux et al., IEEE Journal of Quantum Electronics, 5 (1968) 360.

8. J.L. Champetier et al., C. R. Acad. Sc. Paris, 266 (1968) 838

9- Dispositif présenté dans : J.L. Bobin et al., Nuclear Fusion 9 (1969) 115.

10 Dont : F. Floux et al., Phys. Rev. 1A (1970) 821 ; J. L. Bobin et F. Floux, Rapport CEA R-4255 (1971).

11· J. Nuckolls et al., Physics Today, 26 (1973) 46 ; K.A. Brueckner et S. Jorna, Rev. Mod. Phys. 46 (1974) 325.

12• www-Imj.cea.fr 